\title{
dspace.vutbr.cz
}

\section{Simulink generated control algorithm for nine-phase PMS motor}

\author{
KOZOVSKÝ, M.; BLAHA, P.
}

Proceedings of the 7th IEEE International Conference on Control System, Computing and Engineering (ICCSCE 2017)

pp. 69-74

elSBN: 978-1-5386-3897-2

DOI: http://dx.doi.org/10.1109/ICCSCE.2017.8284380

Accepted manuscript

(C2017 IEEE. Personal use of this material is permitted. Permission from IEEE must be obtained for all other uses, in any current or future media, including reprinting/republishing this material for advertising or promotional purposes, creating new collective works, for resale or redistribution to servers or lists, or reuse of any copyrighted component of this work in other works. Matus Kozovsky, Petr Blaha, " Simulink generated control algorithm for nine-phase PMS motor ", Proceedings of the 7th IEEE International Conference on Control System, Computing and Engineering (ICCSCE 2017), pp. 69-74, 2017. DOI: 10.1109/ICCSCE.2017.8284380. Final version is available at http://ieeexplore.ieee.org/document/8284380/ 


\title{
Simulink generated control algorithm for nine-phase PMS motor
}

\author{
Matus Kozovsky and Petr Blaha \\ CEITEC BUT \\ Brno University of Technology \\ Purkynova 123, Brno 612 00, Czech Republic \\ Phone: +420 541146454 \\ Emails: matus.kozovsky@ceitec.vutbr.cz, petr.blaha@ceitec.vutbr.cz \\ URL: http://www.ceitec.eu/
}

\begin{abstract}
This paper deals with the nine-phase motor control algorithm development, mainly with its implementation in target hardware using MATLAB Simulink. This environment allows control algorithm design using model based approach and the subsequent automatic conversion of control algorithms into C code using Simulink Embedded coder which is then used in the inverter software. Field oriented control algorithm has been designed and tuned for this motor. Control algorithm has been tuned according to the measured motor parameters in simulations and then fine tuned on a real motor. The inverter control unit is based on AURIX Tricore microcontroller TC275. The code generation in Simulink has many settings. This paper analyses computational time depending on these settings. Potential problems with floating point math functions are outlined. Automatic code generation proves to be quick and easy tool for replacement of control algorithms or for fast control algorithm adaptation to different hardware.
\end{abstract}

Index Terms - control algorithms, Simulink, code generation, multi-phase motor, permanent magnet synchronous motor.

\section{INTRODUCTION}

Nowadays, rapid prototyping tools are widely used for the development of new control methods and their tuning in different fields. Many XiL (X-in-the-Loop) tools for rapid prototyping exist. Their functionality is very diverse depending on their use, see [1], [2] and [3], in individual stages of controller development.

The utilization of rapid prototyping tools significantly reduces the time required to create a new controller, as well as the time required for their tuning and debugging. It is very helpful when control algorithm variables can be visualized in real-time and various control algorithm parameters can be adjusted during control algorithm run time. Specialized hardware and software, for example ControlDesk in connection with DCI-GSI2 platform from dSPACE, can be used for this purpose. It enables to monitor and tune variables through debugging connector without necessity to make changes is

The research was supported by ECSEL JU under the project 662192 3Ccar Integrated Components for Complexity Control in affordable electrified cars and by FEKT/FSI-J-16-3694.

The completion of this paper was made possible by the grant No. FEKTS-17-4234 - Industry 4.0 in automation and cybernetics financially supported by the Internal science fund of Brno University of Technology.

The research results were verifed in simulation using AVL CRUISE M simulation SW provided by AVL within University Partnership Program. created code. The reduction of processor power is very low. This approach is sometimes called real-time debugging.

This paper describes steps to develop FOC (Field Oriented Control) algorithm for the nine-phase motor. The main effort of this work is to implement vector control algorithms developed and tested in MATLAB/Simulink environment in AURIX TC275 target. Vector control algorithms have different structures. Some structures are suitable for one multi-phase system (see [4]) while others are developed for multiple threephase systems, considering mutual coupling between subsystems. See [5], [4].

The control loop in MATLAB/Simulink consists of both, the motor model and the control algorithm. Control algorithm parameters can be tuned in this configuration. Various optimization methods can be used to configure control algorithms. The whole process of optimization can be automated using minimization of specific criteria function. Such an automated tuning methods often work iteratively. These methods change parameters of the control loop until they reach minimum of criteria function. Simulation conditions must be the same for every iteration cycle of minimization process. Properly selected simulation conditions are necessary for the correct result of minimization method. Criteria functions can be calculated for example from speed deviation from setpoint, see [6]. They can also have several inputs with different weight.

Code generation and its correct configuration is another important step in the implementation of the control algorithm to the target hardware. This process can be realized manually by rewriting logic structure of control blocks to $\mathrm{C}$ code. However, manual coding is time consuming and mistakes occur very often during this process. On the other hand, the automatic code generation process is often condemned as not optimal due to its high computational demands. Nevertheless using suitable modeling approach along with proper code generation settings can produce fast and comprehensible code. MATLAB/Simulink allows code generation to $\mathrm{C}, \mathrm{C}++$ or VHDL/Verilog languages (with the help of additional toolboxes).

This paper deals with the settings for C language code generation in the environment of MATLAB/Simulink. The difference in computing time with respect to code generation 
settings is analysed. The whole computation time of the control algorithm is tightly connected with target hardware. The difference is not only in the performance of this processor but also in the functions it supports.

\section{CONTROL ALGORITHM DESIGN}

For the design and tuning of the controller, it is necessary to simulate the whole control loop. Control loop is composed from control blocks, motor model, inverter model and auxiliary block (for example conversion of data types, or adaptation of sampling periods). The controller can be designed using knowledge of the controlled system. In our case, it is a ninephase motor (in triple three-phase configuration). The control loop can be seen in Figure 1.

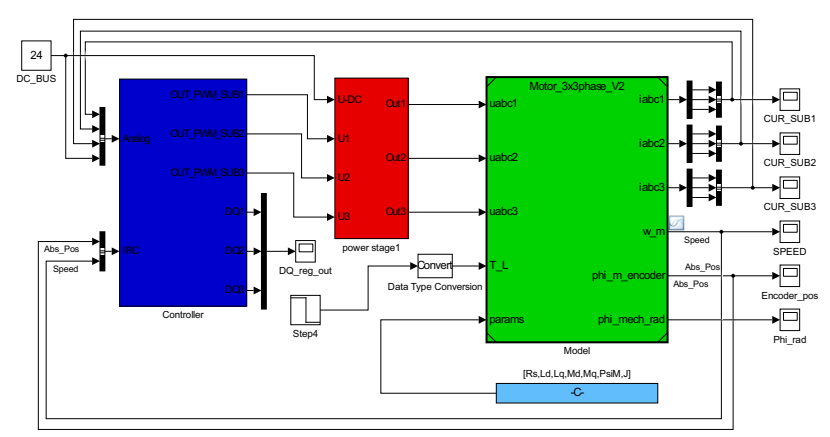

Fig. 1. Complete control scheme.

\section{A. Nine phase PMS motor model}

The nine-phase PMS motor model is created based on the knowledge of motor differential equations [7] and its derivation for nine-phase motor is described in details in [8]. It is based on fundamental equations of the three-phase motor which are extended with respect to mutual relationships between sub-systems.

The behavior of electrical part of the motor can be described in $d q$ coordinates using nonlinear state space representation as follows

$$
\left(\begin{array}{l}
\dot{i}_{d 1} \\
\dot{i}_{q 1} \\
\dot{i}_{d 2} \\
\dot{i}_{q 2} \\
\dot{i}_{d 3} \\
\dot{i}_{q 3}
\end{array}\right)=\boldsymbol{A}_{i}\left(\omega_{e}\right)\left(\begin{array}{l}
i_{d 1} \\
i_{q 1} \\
i_{d 2} \\
i_{q 2} \\
i_{d 3} \\
i_{q 3}
\end{array}\right)+\boldsymbol{B}\left(\begin{array}{l}
v_{d 1} \\
v_{q 1} \\
v_{d 2} \\
v_{q 2} \\
v_{d 3} \\
v_{q 3}
\end{array}\right)+\boldsymbol{A}_{\Psi}\left(\begin{array}{c}
\Psi_{M} \\
0 \\
\Psi_{M} \\
0 \\
\Psi_{M} \\
0
\end{array}\right)
$$

where $i_{x y}$ and $v_{x y}$ are stator currents and voltages respectively, in either $d$ or $q$ axis in one of the three sub-systems. $\Psi_{M}$ is the magnetic flux of permanent magnet in rotor. Matrices $\boldsymbol{A}_{i}\left(\omega_{e}\right)$, $\boldsymbol{B}$ and $\boldsymbol{A}_{\Psi}$ are based on motor parameters and they can be found in [8].

The resulting formula for calculating the torque produced by the motor is given by

$$
\begin{aligned}
T_{m o t}= & \frac{3}{2} p\left[\Psi_{M}\left(i_{q 1}+i_{q 2}+i_{q 3}\right)+\right. \\
& +\left(i_{d 1} i_{q 1}+i_{d 2} i_{q 2}+i_{d 3} i_{q 3}\right)\left(L_{d}-L_{q}\right)+ \\
& +\left(i_{d 1} i_{q 2}+i_{d 2} i_{q 1}+i_{d 1} i_{q 3}+\right. \\
& \left.\left.+i_{d 3} i_{q 1}+i_{d 2} i_{q 3}+i_{d 3} i_{q 2}\right)\left(M_{d}-M_{q}\right)\right]
\end{aligned}
$$

where $L_{d}, L_{q}, M_{d}$ and $M_{q}$ are self inductances and mutual inductances in $d$ and $q$ coordinates and $p$ is the number of pole pairs.

The mechanical part of the motor model is responsible for the conversion of the motor torque $T_{m o t}$ to the speed and the position. The angular acceleration $\varepsilon_{e}$ of the rotor is time derivative of electrical speed $\omega_{e}$ and it depends on moment of inertia $J$ and on the overall torque connected to the rotor. The overall torque is given as a sum of the motor torque $T_{m o t}$, the load torque $T_{\text {load }}$ and the friction torque $T_{\text {fric }}$

$$
\varepsilon_{e}=\frac{d \omega_{e}}{d t}=\frac{d^{2} \varphi_{e}}{d t^{2}}=\frac{1}{J}\left(T_{m o t}+T_{\text {load }}+T_{\text {fric }}\right)
$$

The model in $d q$ coordinates is simpler than the one in $\alpha \beta$ or $A B C$ coordinates because the inductances does not depend on rotor angle. But the coordinate transformations from $A B C$ to $d q$ where the modelling is realized and back to $A B C$ coordinates must be employed using Park and Clarke transformations and their inverses [9] to have a model which corresponds with its inputs and outputs to reality.

\section{B. Controller design}

Having motor model, it is possible to start with controller design. Field oriented control was taken as basis due to its favourable properties. This type of control operates with quantities which are transformed to coordinate system which is fixed to the rotor (in $d q$ coordinates). Park and Clarke transformations are used to convert $A B C$ currents into $d q$ coordinates as in the motor model. Inverse transformations are also used. This method converts harmonic signals which are seen in stationary reference frame to constants in synchronously rotating reference frame which is fixed to the rotor position.

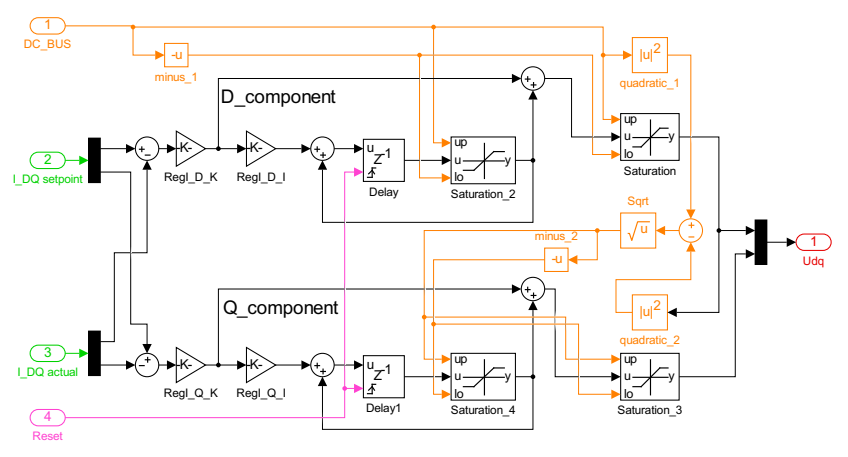

Fig. 2. Internal structure of field oriented controller for one subsystem. 
PI controller is used for current control. In fact, two PI controllers are used for every sub-system (see Fig. 2). One controller is used for the $d$ component of the current and the second one for the $q$ component. Controller structure for $d$ component is defined by equation 4 .

$$
\begin{aligned}
& e_{d}(k)=I_{d_{\text {ref }}}(k)-I_{d_{m}}(k) \\
& v_{d}(k)=K_{P}\left(e_{d}(k)+\frac{T_{s}}{T_{i}} \sum_{n=1}^{k-1} e_{d}(n)\right)
\end{aligned}
$$

Variable $e_{d}(k)$ represents control error, the current reference is represented by $I_{d_{r e f}}(k)$ and $I_{d_{m}}(k)$ is the actual measured value of current recalculated to $d$ axis. Controller structure for $q$ component is defined analogously.

Both controllers outputs are saturated to maximum allowed voltage. Controller output voltage vector magnitude cannot exceed the voltage limit based on DC bus voltage $V_{D C}$ and selected PWM type. Presented control algorithm uses space vector modulation (SVM). Voltage limit is given by equations 5 .

$$
V_{\max }=\frac{V_{D C}}{\sqrt{3}}
$$

$V_{\max }$ is maximum allowed magnitude of voltage vector in $d q$ coordinates.

$d$ component of the controller output signal $v_{d}$ is saturated to $V_{\text {max }}$. Saturation value of $v_{q}$ is given by equation 6 . Magnitude of the voltage vector is lower or equal to $V_{\max }$.

$$
V_{q_{\max }}=\sqrt{{V_{\max }{ }^{2}-v_{d}^{2}}^{2}}
$$

The saturated voltage vector in $d q$ coordinates is then recalculated to stationary reference frame and SVM is used to compute duty cycles for the inverter. The current references can be set manually depending on the required torque or it can be provided by the outer speed control loop.

The overall control scheme contains three field oriented controllers, each for one three-phase subsystem as can be seen in Fig. 3.

Controller designed using MATLAB/Simulink can be easily modified and extended. For example, dead-time compensation block and decoupling block which cancels mutual coupling between sub-systems can be added.

The advantage of this approach also lies in the simplicity of any adjustments. For example, the controller can be reused to work with six-phase motor easily by removing one unused control sub-system and its connections.

\section{FRAMEWORK FOR GENERATED CODE}

The basic framework which needs to be developed before automatic code generation process encompasses service of peripherals required for motor control. These are namely VADC (Versatile Analog to Digital Converter) module for current measurements, GTM (Generic Timer Module) module for PWM (Pulse Width Modulation) signals generation and GPTU (General Purpose Timer Unit) for position detection using incremental encoder signal processing. An important part of the framework is their mutual synchronization. VADC and GPTU need to by synchronized with GTM module. Convenient instant for current measurements depends on current sensing method. Shunt resistors are used in our case. Measurement can thus take place only if all (or at least two) bottom transistors are switched on. GTM module generates the trigger signal for VADC module at the start up of every PWM period. VADC module subsequently generates interrupt on end of conversion. This interrupt reads rotor position from GPTU and it is ready to call generated control algorithms. Computed control action is than stored in shadow registers of GTM module for future center aligned PWM pattern generation. This must be accomplished before actual PWM period ends up. The frequency of PWM was selected to be $10 \mathrm{kHz}$ in our case. This means that the time available for framework execution is 100 $\mu \mathrm{s}$.

The peripherals are configured and serviced using iLLD (Infineon Low Level Drivers). Their utilization is straightforward and reduces time required to study peripherals on a level of control registers.

\section{A. Current measurements}

The time consumed by nine analog to digital conversions, reading analog values and converting them to different formats needs to be taken into account. The VADC module in TC275 contains 8 analog inputs which are collected in two groups of four inputs which can be converted simultaneously. Two sequential conversions are used to convert all required inputs. Result registers contain n-bit unsigned fixed point number depending on selected ADC precision and mode of operation. These numbers must be recalculated to physical quantities according to the measurement ranges. Such prepared values can be used in control algorithm. The process of converting, reading and preparing analog values takes approximately 6 $\mu s$. This time could be partially reduced using DMA (Direct Memory Access) which was not implemented in this work. This time is marked with green color in Fig. 6.

\section{B. Update of PWM duty cycles}

TOM (Timer Output Module) channels which are part of GTM are used for multi-phase center aligned PWM pattern generation. TOM channels can be easily chained together. The first channel usually provides PWM period signal which triggers consecutive channels in the chain. Each TOM channel contains shadow registers which contain information about duty cycles. They are automatically reloaded to action registers when new PWM period starts.

PWM duty cycles must be in specific range. Some minimum nonzero time is necessary due to the current measurement process to avoid sampling of analog values when power transistors are switching. The nine-phase inverter requires eighteen individual PWM channels. Complementary pairs must be equipped with dead-time. There is no hardware support available to insert dead-time automatically in GTM of TC275. Dead-times therefore need to be calculated and inserted via software. Checking duty cycles range, inserting dead-time and 


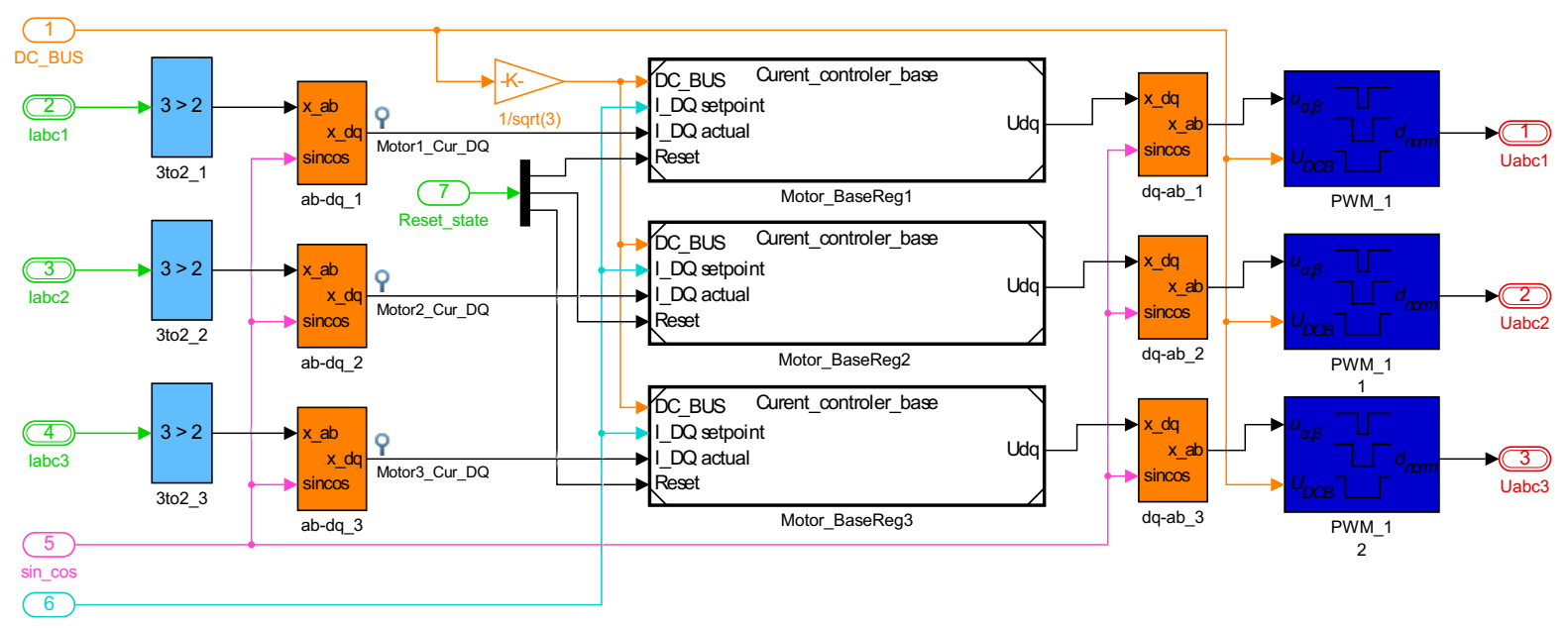

Fig. 3. The overall controller model

writing new values into shadow registers take $13.8 \mu \mathrm{s}$. This time is marked with yellow color in Fig. 6.

\section{Rotor position measurement}

Incremental encoder outputs are connected to GPTU module (GPT12). The signals are treated automatically by the module itself and does not represent computational burden on microcontroller. Actual rotor position and speed are obtained by reading corresponding registers with some conditioning. Required computing time is negligible comparing with previous peripherals.

\section{Automatic CODE GENERATION FROM SimUlink SCHEME}

It is very important to adapt code generation settings to the actual target hardware and also to the compiler. Let's demonstrate this importance on a following example.

Our target microcontroller is AURIX Tricore TC275. This microcontroller has three cores and each core contains Floating Point Unit (FPU) which executes single precision IEE-754 compatible floating point operations. Therefore it is necessary to guarantee that all the computation is realized using single precision floating point operations. In other case, computation is realized using software library and consumes very long time. There are two options how it can be done. Either to define all inputs and all constants as single or by defining largest atomic size of floating-point to float. First approach is used in our work. Default code generation parameters were used with system target file ert.tlc and C language generation. MATLAB version 2015b was used for simulations and code generation.

Free TriCore Entry Tool Chain was used for generating elf files. It uses Hightec GNU compiler and linker. Total computing time for one control algorithm cycle was approximately $163 \mu \mathrm{s}$. Processing time exceeds maximum allowed time given by PWM period. This code generation configuration is not suitable for real-time operation. After analysing generated code it was found out that such a settings used standard mathematical library for all advanced functions as sin, cos, sqrt and others but in 64 bit precision even thought the inputs and outputs were define as 32 bit floats. Code profiling showed that the most time consuming functions were sin and cos. Computation time of sine itself has taken $45 \mu \mathrm{s}$ and computation of sqare root $6.5 \mu \mathrm{s}$. Generated code looks as folows.

test_Y.Out1 = $($ real32_T $) \sin ($ test_U.In 1$)$;

Many experiments with configuring code generation settings were done. The computing time was reduced significantly when standard math library was changed from C89/C90 (ANSI) to C99 (ISO). This change resulted in utilization of single precision functions as follows

test_Y.Out1= sinf(test_U.In 1$)$;

The MATLAB/Simulink provides possibility to use code replacement library. It enables to replace functions and operators in generated code easily by defining custom replacement library with Code Replacement Tool. Custom library is prepared in $\mathrm{C}$ code. User defined function must have the same input and output data types as replaced function. Code generation can then provide following code

test_Y.Out1 = mysinf(test_U.In 1);

where mysinf is custom function for computing sinus function.

\section{A. Custom trigonometric functions}

Different approximations can be used to speed up the computation of sine and cosine functions which are required in FOC algorithm by Park transformation. Figure 4 shows comparison of sinf function computation time with polynomial approximation and with approximation using look-up table. It also shows comparison of relative error taking result from sinf 


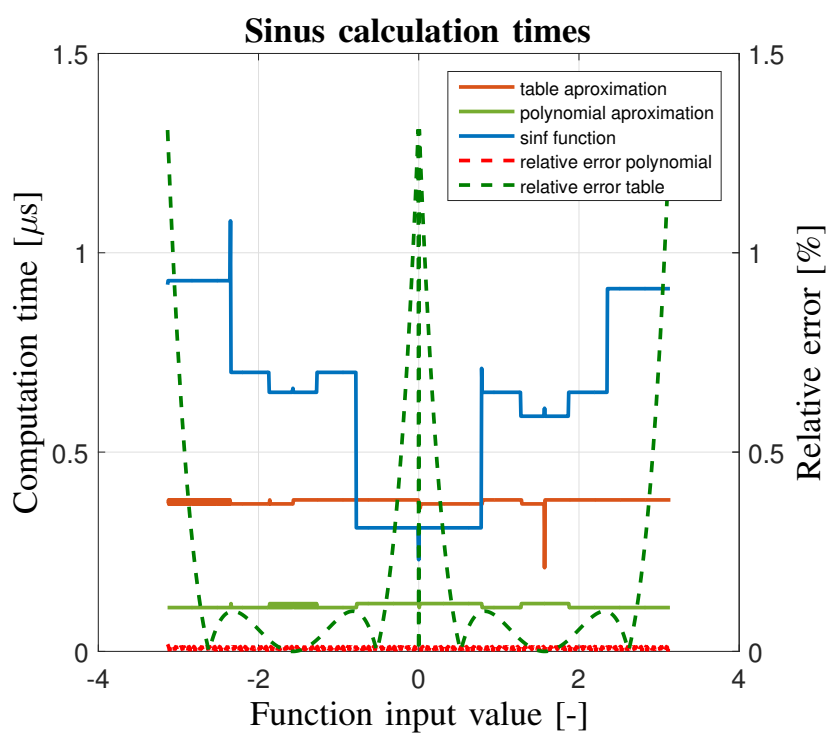

Fig. 4. Computation time and relative error of several sine function approximations

as a reference. Both comparisons are computed for different input angles in the range of $(-\pi, \pi)$.

One can observe that the computation time of sinf function is not constat for different inputs and changes between $(0.3,1.1) \mu s$. This behavior is not very well suited for real time implementation.

Some processors have special functions to calculate trigonometric functions using internal lookup table. This solution can be also implemented in the user library. The lookup table with 64 elements containing one quadrant of sine function and linear approximation between samples is used. Computation time is around $0.37 \mu \mathrm{s}$. Lookup table defining the function in all four quadrants would lead to faster computation for the price of higher memory requirements. Simulink offers block with this functionality between lookup table blocks.

The next possibility is to use polynomial approximation. Besides Taylor series approximation there exist fast and accurate approximation of sine function as follows

$$
\begin{array}{r}
y=\frac{4}{\pi} x-\frac{4}{\pi^{2}} x|x| \\
\sin x \approx P(y|y|-y)+y
\end{array}
$$

where $P=0.225$ is the optimal weight for minimizing absolute error (this value was used in our experiments) and $P=$ 0.218 is optimal value for minimizing relative error. Computation time is around $0.12 \mu \mathrm{s}$. The relative error depends on input angle but it is mostly below $0.12 \%$. Computation of cosine function is based on equality $\cos (x)=\sin (x+\pi / 2)$.

\section{B. Custom sqrt function}

Custom replacement can also be applied for other functions. Another computationally demanding mathematical function used in FOC algorithm is square root. It is used for voltage constraint computation of PI controllers. The precision is not critical in this case. Figure 5 shows comparison of sqrtf function computation time with recursive $6^{\text {th }}$ order Newton's

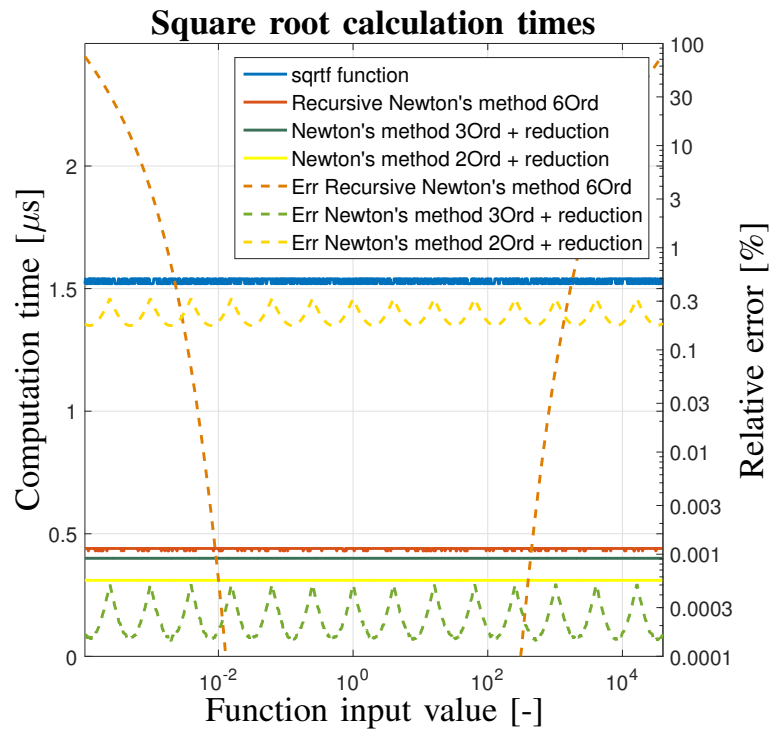

Fig. 5. Computation time and relative error of several square root approximations

method and with two Newton's methods with reduction $\left(2^{\text {nd }}\right.$ and $3^{\text {rd }}$ order). Calculation time is reduced from $1.5 \mu \mathrm{s}$ for sqrtf function down to $0.3 \mu s$ for $2^{\text {nd }}$ order Newton's methods with reduction. Relative error for the fastest version is below $0.3 \%$ which is acceptable.

\section{Enable signal store reuse}

Automatic code generation process allocates a separate memory buffer for each block's outputs without this option. This makes all block outputs global and unique, which in many cases significantly increases RAM and ROM usage. This feature is suitable for monitoring program run in realtime. Reduced RAM usage can also reduce computation time during the optimization process. Signals which are important for debugging and tuning process can be configured as test points. Test points are always global and can be added to any signal in Simulink scheme. All test points can be ignored during code generation process. This feature is suitable for final implementation.

\section{Enable local block output}

Specify whether block output signals are declared locally or globally. Locally declared block output signals are not visible using global addresses. Standard rapid prototyping tools operate with global variables. All blocks variables used in control scheme are still tunable. The structure of all parameters is stored in global memory. Computing time having this option on is reduced to $26.2 \mu \mathrm{s}$.

\section{E. Enable inline parameters}

Enable inline parameters function option converts all variables in Simulink blocks to constants. This code is then not tunable because all variables are stored in program memory code as constants. This is a suitable situation for some blocks. Computing time reduction depends primarily on the number of blocks containing tunable parameters. For example, change 
of sign can be realized by gain block with the constant value of -1 . However, this constant does not have to be tunable and the same operation can be realized using unary minus block.

The best solution is to use Simulink parameters. Several code generation options are available for this data type. Simulink parameters can be connected to any extern variable of the prepared framework. The Simulink parameter can be configured as tunable even thought inline parameters are enabled.

Computing time of control algorithm is reduced to $22.4 \mu \mathrm{s}$ using all previous optimization methods and inline parameters. Total computing time with the framework is approximately $42.2 \mu s$.

The influence of individual optimizations and code generation settings is summarized in Table I and in Fig. 6.

TABLE I

CONTROL ALGORITHMS EXECUTION TIMES

\begin{tabular}{|l|c|}
\hline Variant of optimization & $\begin{array}{c}\text { Computing } \\
\text { time }[\mu \mathrm{s}]\end{array}$ \\
\hline VADC result registers loading and convert to float & 6 \\
\hline Writing PWM dutys to TOM registers & 13.8 \\
\hline V1. Without optimization using 64 bits math functions & 163.2 \\
\hline V2. Without optimization using 32 bits math functions & 44.8 \\
\hline V3. Custom sin, cos and square root & 40.2 \\
\hline V4. Enable signal store reuse & 38.2 \\
\hline V5. Reuse local, global block and eliminate superfluous & 36.5 \\
\hline V6. Enable local block output & 26.2 \\
\hline V7. Enable inline parameters & 22.4 \\
\hline
\end{tabular}

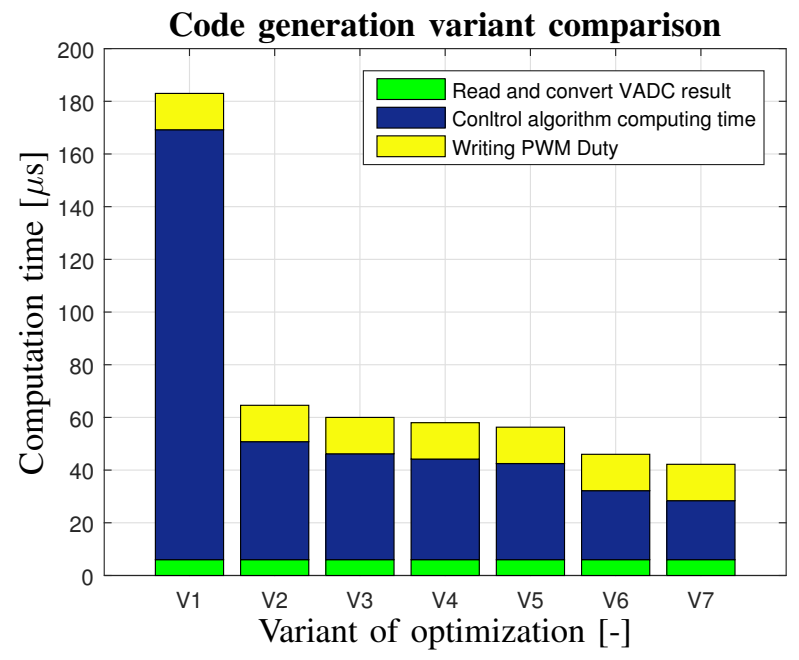

Fig. 6. Control algorithms execution times

\section{CONCLUSION}

Automatically generated control algorithm can be used to control triple three-phase motor on AURIX Tricore TC275 microcontroller. Control algorithm tuning and debugging process can be easily realized using measurement and calibration tools (for example dSPACE platform). Modification of control structure can be easily realized due to employed method of automatic code generation.
The automatically generated code is fast enough for practical use. Microcontroller load using all optimizations is about $42 \%$ using $10 \mathrm{kHz}$ PWM period. The control algorithm can be distributed into all three cores in the case of higher computational requirements. The manually written code could achieve higher performance, however tuning and debugging process of the entire system would be extremely time consuming. Demonstrated automatic code generation process is suitable for simple and fast preparation of control algorithm structure. This solution is also suitable for developing special control features.

The additional fine tuning in real hardware was realized since the model and the real system were not in perfect coincidence in all situations. This monitoring and calibration was achieved using dSPACE DCI-GSI2 system.

\section{REFERENCES}

[1] P. Blaha and P. Vaclavek, "Rapid prototyping of robust motor control algorithms on freescale targets," in Proceedings of the 2013 IEEE/SICE International Symposium on System Integration. IEEE, Dec. 2013, pp. 629-634.

[2] F. E. H. Velasco, N. T. Garcia, and F. A. Garcia, "Rapid Control Prototyping of a permanent magnet DC motor using non-linear sliding control ZAD and FPIC," in 2012 IEEE 3rd Latin American Symposium on Circuits and Systems (LASCAS). IEEE, Feb. 2012, pp. 1-4.

[3] K. Meah, S. Hietpas, and S. Ula, "Rapid Control Prototyping of a Permanent Magnet DC Motor Drive System using dSPACE and Mathworks Simulink," in APEC 07 - Twenty-Second Annual IEEE Applied Power Electronics Conference and Exposition. IEEE, Feb. 2007, pp. 856-861.

[4] A. S. Abdel-Khalik, A. Elserougi, S. Ahmed, and A. Massoud, "Vector control of multiphase induction machine under open-circuit phase faults," in 4th International Conference on Power Engineering, Energy and Electrical Drives. IEEE, may 2013, pp. 229-234. [Online]. Available: http://ieeexplore.ieee.org/document/6635611/

[5] G. Singh, K. Nam, and S. Lim, "A Simple Indirect Field-Oriented Control Scheme for Multiphase Induction Machine," IEEE Transactions on Industrial Electronics, vol. 52, no. 4, pp. 1177-1184, aug 2005. [Online]. Available: http://ieeexplore.ieee.org/document/1490709/

[6] S. Lavania and D. Nagaria, "Fminsearch Optimization Based Model Order Reduction," in 2016 Second International Conference on Computational Intelligence \& Communication Technology (CICT). IEEE, feb 2016, pp. 568-571. [Online]. Available: http://ieeexplore.ieee.org/document/7546672/

[7] T. Miller and M. McGilp, "Analysis of multi-phase permanent-magnet synchronous machines," in 2009 International Conference on Electrical Machines and Systems. IEEE, Nov. 2009, pp. 1-6.

[8] M. Kozovsky, P. Blaha, and P. Vaclavek, "Verification of nine-phase PMSM model in d-q coordinates with mutual couplings," in 2016 6th IEEE International Conference on Control System, Computing and Engineering (ICCSCE). IEEE, 2016, pp. 73-78. [Online]. Available: http://ieeexplore.ieee.org/document/7893548/

[9] Z. Wen and S. He, "Analysis of three-phase magnitude-phase detection method based on double dq transformation," in Proceedings of The 7th International Power Electronics and Motion Control Conference. IEEE, jun 2012, pp. 340-344. [Online]. Available: http://ieeexplore.ieee.org/document/6258869/ 